\title{
Improvements to the HNR Estimation Based-on Generalized Variogram
}

\author{
Diana Torres-Boza* and Carlos A. Ferrer \\ Center for Studies on Electronic and Information Technologies, Central University \\ Marta Abreu of Las Villas, Carretera de Camajuaí Km 5 1⁄2 Santa Clara Villa Clara, Cuba \\ $\{d t b, c f e r r e r\} @ u c l v . e d u . c u$
}

\begin{abstract}
The presence of an unusual high level of turbulent noise in voice signals is related to air leakage in the glottis as a result of incomplete closure of the vocal cords. Harmonics to Noise Ratio (HNR) is an acoustic measure that intends to appraise the amount of that turbulent noise. Several algorithms have been proposed in both time and frequency domain to estimate HNR. The Generalized Variogram (GV) is a time-domain technique proposed for HNR estimation based on a similitude function between two speech windows. The drawbacks of the GV are related to the biased estimation of the amplitude ratio and the final HNR value. The present work deals with these limitations and proposes unbiased estimators. The experimental results show that the described improvements outperform the original GV.
\end{abstract}

Keywords: harmonics to noise ratio, additive noise, shimmer, variogram.

\section{Introduction}

Acoustic measures are a widely used tool to help speech pathologists diagnosing and documenting treatment and therapy progress of laryngeal diseases. Several acoustic measures related to vocal cords diseases have been reported through the years.

Healthy vocal cords produce a quasi-periodic vibration pattern but in dysphonic voices a deviation from this cycle-to-cycle periodicity appears [1]. Pitch perturbation (vocal jitter) is defined as the cycle-to-cycle fundamental frequency variations, amplitude perturbation (vocal shimmer) is defined as the cycle-to cycle amplitude variations, and the additive noise is related to the turbulent noise produced at the glottis and is correlated with the degree of dysphonia [2][4]. This paper is specifically aimed for the improvement of an acoustic measure of additive noise.

Harmonics to Noise Ratio (HNR) is an acoustic measure of the amount of additive noise that appears during the vibration of the glottis [1]. This noise is produced by an air leakage through the vocal cords caused by the presence of pathologies such as nodules, polyps and cancer, among others [1]. HNR estimation can be achieved in

\footnotetext{
* Corresponding author.
} 
both time and frequency domains. Frequency domain algorithms require the estimation of the harmonic structure, usually by means of Fourier Transform, and it has been criticized for the impossibility to separate measurements of different types of perturbations [5]. Time domain methods have been criticized [3][6][7][8][9] for depending on the correct determination of individual pulse boundaries, among many other method-specific factors. In spite of the latter criticism, this work is specifically centered in time domain HNR estimation, due to the advantage over frequency domain based methods to separate different perturbations.

Reference [9] proposes an HRN estimation technique, based on generalized variogram $(\mathrm{GV})$, that takes into account the non-stationary nature of voice signals and the limitations of boundaries estimation while facing pathological voices. One of the advantages of this method is its ability to estimate the HNR values independently from the temporal or spectral structures of voice signals. The principal limitations are the biased estimation of the gain factor and the influence of the noise component in the harmonic part in the HNR estimator. Based on this, the main goal of this work is to correct these limitations and to evaluate its performance compare not only to the original estimator but to other well-known algorithm proposed in the literature [11].

\subsection{HNR Based on Generalized Variogram}

For stationary time series the variogram is directly related to the auto covariance function [10]. In [9] the GV is proposed taking into account the properties of the speech signal.

Let $x(n)$ denote a pseudo-periodic signal for some $T_{0}$ :

$$
x(n)=a_{k} x\left(n-k T_{0}\right), \quad k=0,1,2, \ldots
$$

Where $a_{k}$ is the weighting coefficient and it is related with amplitude changes in the speech signal, $T_{0}$ is the length of the glottal cycle and represents the fundamental period.

The formulation of HNR for a voice segment $x(n)$ in $\mathrm{dB}$ is given by the expression:

$$
H N R_{x}(d B)=10 \log \left[\frac{\sum_{n=0}^{N-1} \tilde{x}(n)^{2}}{\sum_{n=0}^{N-1} e(n)^{2}}\right]
$$

Where the numerator represents the energy of the harmonic component and the denominator is related with the energy of the additive noise component in $x(n)$.

To estimate the energy of the amount of noise (denominator in (2)) the following expression was proposed in [9]:

$$
\operatorname{Den}=\min _{T}\left[\sum_{n=1}^{N-1}(x(n)-a x(n-T))^{2}\right], \quad-T_{\max }<T<-T_{\min } \text { and } T_{\min }<T<T_{\max }
$$


The expression for $\mathrm{GV}$ is within the brackets in (3). $N$ is the frame length in samples (chosen as $2.5 \mathrm{~ms}$ ) and $T$ is related to the glottal pulse length. Since GV is computed in forward and backward directions, $T$ lies within the intervals shown in (3). $T_{\min }$ and $T_{\max }$ represent the shortest and longest suitable glottal cycle length in samples respectively. These values were fitted to $2.5 \mathrm{~ms}$ and $20 \mathrm{~ms}$ [2].

The maximum similarity between $x(n)$ and the lagged frame $x(n-T)$ is achieved when expression (3) reaches the minimum value.

The expression for $a$ is defined as:

$$
a=\sqrt{\frac{E_{x(n)}}{E_{x(n-T)}}}
$$

Where $E_{x(n)}$ and $E_{x(n-T)}$ are the energies at the current and lagged frames respectively.

Having the value of $T\left(T_{\text {opt }}\right.$ ) which minimizes the sum of squared errors (3) and the value of $a$, the noise component in the denominator of $H N R_{x}$ expression is:

$$
e(n)=x(n)-a x\left(n-T_{o p t}\right), 0 \leq n \leq N-1
$$

Therefore the expression for the clean signal can be defined as:

$$
\tilde{x}(n)=x(n)-e(n)
$$

An analysis of the limitations of equations (1), (4), (5) and (6) follows.

The model in (1) does not hold for stationary noise since (1) means that:

$$
\begin{aligned}
\tilde{x}(n)+e_{k}(n) & =a_{k}\left(\tilde{x}\left(n-k T_{0}\right)+e_{k}\left(n-k T_{0}\right)\right), \quad k=1,2,3,4, \ldots \\
& =a_{k} \tilde{x}\left(n-k T_{0}\right)+a_{k} e_{k}\left(n-k T_{0}\right)
\end{aligned}
$$

Where a proportional term $a_{k}$ between lagged noise samples $e_{k}\left(n-k T_{0}\right)$ appear. A more realistic model for the speech signal is given in [12], using the harmonic and noise component terms, as:

$$
\begin{aligned}
& x(n)=\tilde{x}(n)+e_{k}(n), \\
& \tilde{x}(n)=\tilde{a} \dot{x}\left(n-k T_{0}\right), \quad k=1,2,3,4, \ldots
\end{aligned}
$$

From (8) it is possible to obtain an expression for the harmonic component of the shifted frame $\tilde{x}(n-T)$ as:

$$
\tilde{x}(n-T)=\frac{\tilde{x}(n)}{a}
$$


Substituting (8) and (9) in (4) the following expression is obtained:

$$
a^{2}=\frac{\left.\sum \tilde{x}(n)+e_{1}(n)\right)^{2}}{\sum\left(\frac{\tilde{x}(n)}{a}+e_{2}(n)\right)^{2}}=\frac{\sum\left(\tilde{x}(n)^{2}+e_{1}(n)^{2}\right)}{\sum\left(\frac{\tilde{x}(n)^{2}}{a^{2}}+e_{2}(n)^{2}\right)}=a^{2}\left[\frac{\left.\sum \tilde{x}(n)^{2}+e_{1}(n)^{2}\right)}{\left.\sum \tilde{x}(n)^{2}+a^{2} e_{2}(n)^{2}\right)}\right]
$$

As can be observed from (10) the original expression to estimate the gain factor $a$ is biased by the factor within the brackets.

Analyzing the numerator of (2) it is possible to substitute it by (5) and (6) as:

$$
\text { Num }=\sum_{n=1}^{N} \tilde{x}(n)^{2}=\sum_{n=1}^{N}(a x(n-T))^{2}=\sum_{n=1}^{N}\left(\tilde{x}(n)^{2}+a^{2} e(n)^{2}\right)
$$

As can be seen in (11) the numerator of the HNR expression overestimate the real value of HNR as it has a noise term within the numerator. Therefore the original overestimated HNR expression without the log operation can be expressed as:

$$
H N R_{x}=\frac{\sum_{n=0}^{N-1}\left(\tilde{x}(n)^{2}+a^{2} e(n)^{2}\right)}{\sum_{n=0}^{N-1} e(n)^{2}}
$$

\section{Proposed Modifications}

The derivation of factor $a$ is a minimization problem. The $a$ value is optimum when the GV expression (3) reaches its minimum value, so the similarity between the two current periods is maximum.

Substituting (8) in the term within the brackets in (3), it is possible to obtain the following expression for the GV:

$$
G V=\sum_{n=1}^{N-1}(x(n)-a x(n-T))^{2}=\sum_{n=1}^{N-1}\left(e_{1}^{2}(n)+a^{2} e_{2}^{2}(n)\right)=\left(1+a^{2}\right) \sum_{n=1}^{N-1} e^{2}(n)
$$

To obtain $a$, the derivative of (13) respect to $a$ is taken and equated to 0 .

$$
\frac{d G V}{d a}=\left[\frac{G V}{\left(1+a^{2}\right)}\right]^{\prime}=\left[\frac{\sum_{n=1}^{N-1}(x(n)-a x(n-T))^{2}}{1+a^{2}}\right]^{\prime}=0
$$


Developing expression (14) the optimal gain factor $a$ is obtained as:

$$
a=R+\sqrt{1+R^{2}}, \text { where } \mathrm{R}=\frac{x^{2}(n)+x^{2}(n-T)}{2 x(n) x(n-T)}
$$

This obtained value of $a$ is similar the one in [12]. Therefore from (13) the corrected noisy component in (2) is obtained as:

$$
e_{c}^{2}(n)=\min (\text { Den })=\left(\frac{x(n)-a x\left(n-T_{\text {opt }}\right)}{1+a^{2}}\right)^{2}, 0 \leq n \leq N-1
$$

Where the gain factor $a$ can be estimated according to (14).

Having expressions to estimate both the gain factor $a$ and the energy of the noise component $e(n)$ by a corrected variogram, an enhanced expression for HNR can be obtained.

Developing equation (12) we can obtain the following expression to estimate HNR as:

$$
10 \log \left(H N R_{x}-a^{2}\right)=10 \log \left[\frac{\sum_{n=0}^{N-1} \tilde{x}(n)^{2}}{\sum_{n=0}^{N-1} e(n)^{2}}\right]
$$

It will be demonstrated that this expression solves the overestimation of the actual values of HNR.

\subsection{Experiments}

Synthetic signals were generated according to the procedure in [13] and [14] where an all-pole filter with formant frequencies corresponding to a vowel " $a$ " is excited by a train of impulses. As the proposed corrections are related to the amplitude factor $a$, seven levels of both Gaussian noise and shimmer were introduced to meet a desired signal to noise ratio (SNR) and shimmer in the simulated signals. The added values of shimmer and noise used are shown in Table 1.

Table 1. Levels of actual HNR and Shimmer used for the experiments

\begin{tabular}{llllllll}
\hline Level & 1 & 2 & 3 & 4 & 5 & 6 & 7 \\
\hline HNR (dB) & 22 & 18 & 15 & 12 & 8 & 5 & 2 \\
\hline Shimm $(\%)$ & 47.6 & 40.8 & 34 & 27.2 & 20.4 & 13.6 & 6.8 \\
\hline
\end{tabular}

The length of the synthesized signals is $2 \mathrm{~s}$, with sampling frequency Fs $=22050$ $\mathrm{Hz}$ and mean $\mathrm{F}_{0}=150 \mathrm{~Hz}$, giving an average of 300 pulses per signal.

The proposed HNR estimation corrections (GVC) were evaluated by comparing them with the original method $(\mathrm{GV})$ proposed in [8] and with a well-known HNR 
algorithm based on cesptrum estimation (denoted as "Murp") proposed in [11]. Estimated HNR values were also compared with reference values (denoted as "Ref") listed in Table 1 which were added during synthesis of simulation signals.

\section{Results}

Figure 1 shows estimated HNR values for the synthetic vowel contaminated with only amplitude perturbations (shimmer). The $\mathrm{x}$-axis represents the different levels of synthetized shimmer where $6.8 \%$ denotes the lowest level of perturbation and $47.6 \%$ the highest level of contamination. The y-axis represents the estimated values of HNR. In the case of signals synthesized only with shimmer, 30dB of HNR was added to avoid infinite HNR values. The reference values of HNR can be observed in Fig. 1 as an X-dashed line, and the rest of the estimates analyzed as solid lines.

Fig.1 demonstrates the superiority of the GVC over the original GV and the cepstrum-based variants. A biased estimation of the gain factor produces an overestimation of the HNR values facing the shimmered signals. In addition, the inferior performance of $\mathrm{GV}$ respect to $\mathrm{GVC}$ is related to the introduction of the error term in the HNR equation (2). Cepstrum based algorithm shows the worst performance of the three analyzed estimators. As the shimmer cannot be separated from the additive noise in the frequency domain this estimator doesn't produces values related to additive noise alone but to dysperiodicity in a general sense.

The performance of the algorithms using synthesized signals with only additive noise is shown in Fig.2. Unlike the previous figure, the $\mathrm{x}$-axis corresponds to the HNR (in $\mathrm{dB}$ ) actual values and are ordered from the noisiest signals $(2 \mathrm{~dB})$ to the cleanest one $(22 \mathrm{~dB})$. It can be observed from the figure that GVC and "Murp" approaches follow more precisely the dashed line which represents reference values of HNR. Therefore there is superiority in the performance of GVC and "Murp" over the original variogram. The GVC approach removes the influence of the noise term in the HNR formula (2).

Results for signals contaminated with both shimmer and additive noise are shown in Fig.3. It can be seen that also the GVC superiority respect to both "Murp" and original variogram methods.

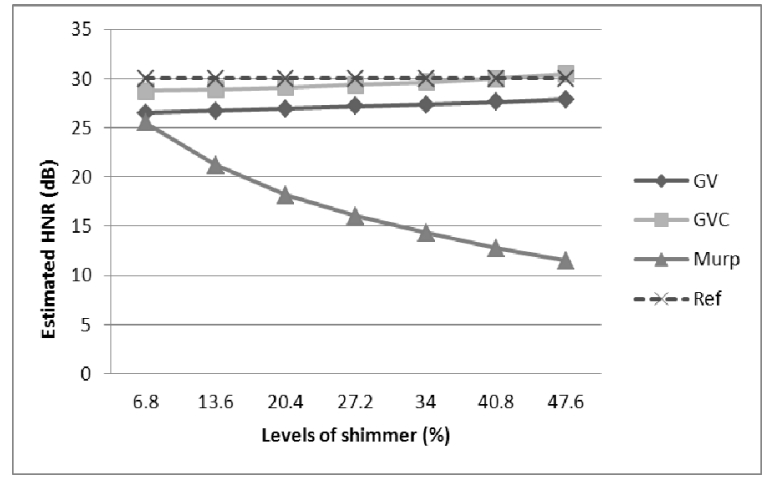

Fig. 1. Results for estimated HNR values for signals contaminated with only shimmer. The dashed line represents the reference values of HNR $(30 \mathrm{~dB})$ for each level of shimmer. 


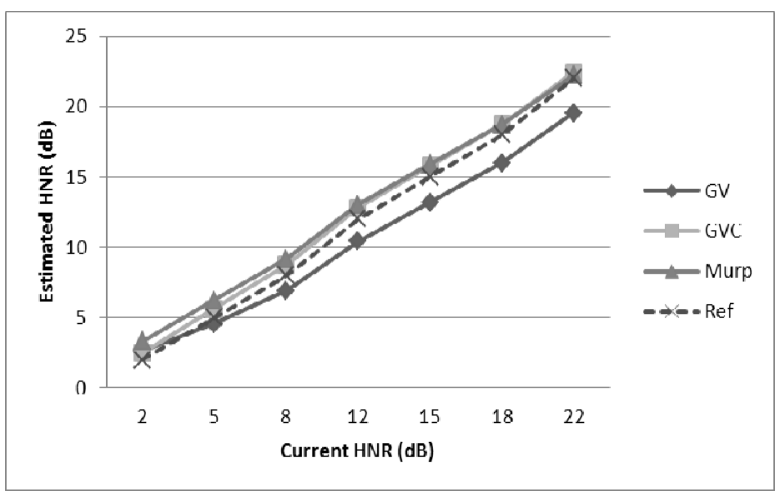

Fig. 2. Results for signals contaminated with only additive noise. The dashed line represents the reference values of HNR for each synthesized level.

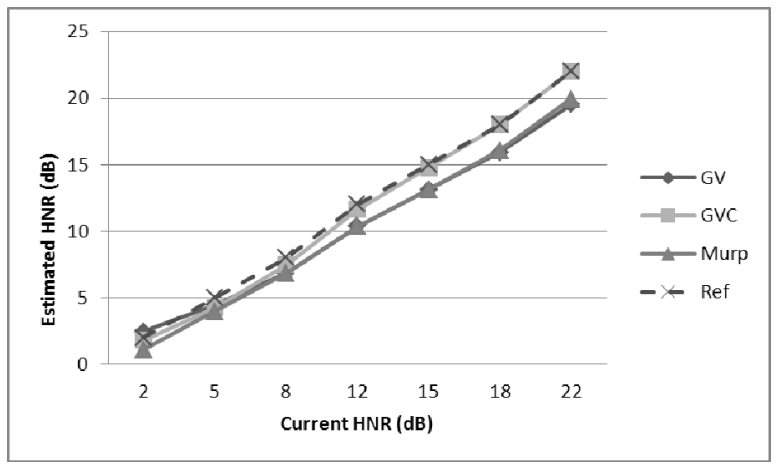

Fig. 3. Results for signals contaminated with both shimmer and additive noise. The dashed line represents the reference values of HNR for each level of perturbations.

\section{Conclusions}

This work is focused on improving the estimation of the Harmonics to Noise Ratio based on the generalized variogram. The experiments results have demonstrated that the original algorithm proposed in [9] overestimates real values of HNR and the gain factor estimator is biased. As expected the best results corresponds to the proposed method, which removes the influence of the noise from the harmonic part of the HNR estimation formula.

\section{References}

1. Hillenbrand, J.A.: Methothological Study of Perturbations and Additive Noise in Synthetically Generated Voice Signals. Journal of Speech and Hearing Research 30, 448461 (1987) 
2. Hillenbrand, J.A.: Perception of aperiodicities in synthetically generated signals. Journal of Speech and Hearing Research 83, 2361-2371 (1988)

3. Hillenbrand, J., Cleveland, R., Erickson, R.: Acoustic Correlates of Breathy Vocal Quality. Journal of Speech and Hearing Research 30, 448-461 (1994)

4. Yumoto, E., Gould, J.W., Baer, T.: Harmonics to Noise ratio as an index of the degree of hoarseness 71, 1544-1550 (1982)

5. Schoengten, J.: Spectral Models of Additive and Modulation Noise in Speech and Phonatory Excitation Signals. Journal of the Acoustical Society of America 113, 553-562 (2003)

6. Qi, Y., Hillman, R.E.: Temporal and spectral estimations of harmonics-to-noise ratio in human voice signals. Journal of the Acoustical Society of America 102, 537-543 (1997)

7. Qi, Y., Hillman, R.E., Milstein, C.: The estimation of signal-to-noise ratio in continuous speech for disordered voices. JASA 105, 2532-2535 (1999)

8. Bettens, F., Grenez, F., Schoengten, J.: Estimation of vocal dysperiodicities in disordered speech by means of distant-sample bidirectional linear predictive analysis. Journal of the Acoustical Society of America 117, 328-334 (2005)

9. Kacha, A., Grenez, F., Schoentgen, J., Benmahammend, K.: Dysphonic Speech Analysis Using Generalized Variogram. In: IEEE International Conference on Acoustics, Speech, and Signal Processing, pp. 917-920. IEEE Press, Philadelphia (2005)

10. Haslett, J.: On the sample variogram and sample autocovariance for non-stationary time series. The Statistician 46(4), 475-485 (1997)

11. Murphy, P., Akande, O.: Quantification of glottal and voiced speech harmonics to noise ratio using cepstral-based estimation. In: ITRW on Nonlinear Speech Processing, pp. 19$22(2005)$

12. Mienkovic, P.: Least mean Squared Error Measures of Voice Perturbations. Journal of Speech and Hearing Research 30, 529-538 (1987)

13. Medan, Y.: Super resolution pitch determination of speech signals. IEEE Transaction on Signal Processing 39, 40-48 (1991)

14. Parsa, V., Jamieson, D.G.: A comparison of high precision F0 extraction algorithms for sustained vowels. Journal of Speech, Language and Hearing Research 42, 112-126 (1999) 